\title{
Elastic-plastic transition: A universal law
}

\author{
Zhong Chen ${ }^{1}$, Hyuk Jong Bong ${ }^{2}$, Dayong Li ${ }^{3}$ and R.H. Wagoner ${ }^{2, a}$ \\ ${ }^{1}$ Research and Innovation Center, Ford Motor Company, Michigan, 48131, United States \\ ${ }^{2}$ Department of Materials Science and Engineering, The Ohio State University, Columbus, 43210, United States \\ ${ }^{3}$ School of Mechanical Engineering, Shanghai Jiao Tong University, 200240, China
}

\begin{abstract}
.
Although the initial stress-strain behavior in a tensile test is often characterized as linear elastic up to a yield stress and nonlinear plastic thereafter, the pre-yield transition region is known to exhibit significant curvature and hysteresis. Hundreds of high-precision loading-unloading-loading tensile tests were performed using 26 commercial sheet alloys exhibiting a wide range of strength, ductility and crystal structure. Analysis of the results reveals the following:

1. There is no significant linear elastic region; the proportional limit is $\sim 0 \mathrm{MPa}$ when measured with sufficient sensitivity.

2. Each of the hundreds of measured transitional stress-strain curves can be characterized by a single parameter, here called the "modulus reduction rate." The corresponding equation captures $\sim 80 \%$ of the observed variation, a factor of 3 to 6 better than a one-parameter linear approximation.

3. Most interestingly, the transitional behavior for all alloys follows a "Universal Law" requiring no fit parameters. The law depends only upon the strength of the material and its Young's modulus, both of which are can be measured by independent tests or adopted from handbooks. The Universal Law captures $\sim 90 \%$ of the variation represented by the one-parameter representation and eliminates the need for mechanical testing to implement and apply.

The practical and theoretical implications of these results are discussed. The results provide a simple path to significantly improving applied constitutive models in the transitional regime. The consistency of the effect for such a wide range of metals and suggests that the origin of the behavior lies in the pile-up and relaxation of dislocation arrays.
\end{abstract}

\section{Introduction}

While it has long been known that mechanical testing produces stress-strain slopes lower than physicallymeasured Young's modulus, the phenomenon was trivialized as a testing problem, or an anelastic rate effect. It was only of passing academic interest until the 1990's when Morestin and Boivin [1] showed that springback after sheet forming occurred according to the mechanically measured, reduced modulus, not the handbook / physically measured value.

Following that discovery, in the 2000's many researchers interested in springback following sheet forming advocated characterizing the behavior as a reduced Young's modulus $(10-30 \%$ reductions were typically reported) in the pre-yield region, and presented results showing that improved simulation accuracy using this approach. [2,3]

In the 2010's, Sun and Wagoner [4] measured the transitional behavior in detail, not only upon loading or unloading, but also in unloading/loading loops, with partial and full cycles, singly and in multiple sequences, and at various rates (none of which were significant to first order). They showed that the deformation was not classically elastic or plastic, nor was it was timedependent anelasticity. Instead, the pre-yield deformation was elastic in the sense that the original shape (no strain) was recovered after a full loop. However, the pre-yield deformation was plastic in the sense that energy was dissipated hysteretically. However, it had a remarkable and mysteriously simple feature: it was quantitatively identical in two distinct alloys having the same strength and Young's modulus even though the strength was attained in divergent ways (e.g. by alloy content / precipitates vs. strain hardening).

Sun and Wagoner proposed a general, 3-D mechanics formulation called QPE (Quasi-Plastic Elastic) that reproduced the transition region according to the observations noted above. It added a third regime of behavior between a purely elastic region and an elasticplastic one. The shape of pre-yield stress-strain function was controlled by up to 4 parameters including the initial slope $\mathrm{E}_{0}$ (if not the Young's modulus) and the strain extent of the linear region (strain proportional limit), $\varepsilon_{\mathrm{c}}$.

Extending Morestin and Boivin's conclusion, Sun and Wagoner also showed that incorporating the nonlinear behavior in FE simulations was essential to predicting springback behavior accurately, i.e. that using a chord modulus, no matter how attractive and convenient, was insufficient. In fact, using the QPE formulation reduced springback error by factors of 2-6 compared with chord modulus and handbook modulus formulations.

The most obvious (but incorrect) candidate mechanism for the modulus effect is related to nonlinear

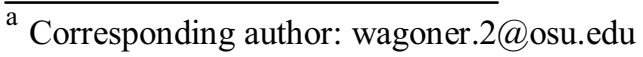


elasticity. An equation proposed for the tangent modulus $\mathrm{E}_{\mathrm{T}}$ [5] based on atomic binding energy curves [6] takes the following form:

$$
E_{T}(\varepsilon)=E_{0}(1-\alpha \varepsilon) \exp (-\alpha \varepsilon)
$$

where $\mathrm{E}_{0}$ is the initial slope (Young's Modulus normally) and $\alpha$ is a material constant. However, atomic bond stretching is purely elastic, i.e. reversible and energypreserving, with no hysteresis. Furthermore, in metals, which yield at small fractions of their theoretical strength, elastic behavior is essentially linear. Therefore, bondstretching can be ruled out as the source of pre-yield nonlinearity.

A simple modification of Eq. (1) to allow for hysteresis within a nonlinear elastic framework was proposed by Zhu et al. [7]. At the same time, the (1- $\alpha$ term) in Eq. (1), which is inconsequential in metals, was removed as a simplification.

Most recently, Chen et al. [8] performed hundreds of tests using 12 diverse steels to identify the range of preyield behavior. They reported the following simplifications that are used in the current work:

- The initial tangential modulus is Young's modulus.

- Loading and unloading nonlinearity are the same, although unloading is preferred for testing because of reduced scatter.

- The only factor altering the pre-yield behavior of 12 steels quantitatively is the strength of the material.

- The transitional behavior is essentially isotropic.

- The transitional behavior is nearly independent of prestrain (larger than $2 \%$ ) except for the effect strain hardening on the strength of the material.

The current work was motivated by questions raised primarily by the Sun and Wagoner [4] and Chen et al. [8] results. The main questions in this study are as follows:

- What is the nature of the elastic-plastic transition? What is the key mechanism induces the nonlinearity?

- Is it possible to represent the nonlinear transition behavior by a simple and single equation?

Based on the foregoing, the extensive testing was streamlined by assuming that the the transition behavior is independent of strain rate, pre-strain (except for prestrain hardening), multiple cycles, direction of testing, loading vs. unloading. In particular, only unloading legs at one prestrain were used.

\section{Theoretical Background}

\subsection{QPE representation}

Sun and Wagoner [4] proposed, developed, and implemented a three-regime general constitutive model. For uniaxial tension, the scalar total strain increment can be decomposed into three parts.

$$
d \varepsilon=d \varepsilon_{e}+d \varepsilon_{p}+d \varepsilon_{Q P E}
$$

where $\mathrm{d} \varepsilon_{\mathrm{e}}$ is the elastic strain, $\mathrm{d} \varepsilon_{\mathrm{p}}$ is the plastic strain and $\mathrm{d} \varepsilon_{\mathrm{QPE}}$ is the so-called QPE strain. Figure 1 shows a typical unloading-loading hysteresis loop originated by the continuous changes of tangential modulus during unloading followed by reloading. The elastic strain is readily calculated from the initial slope (which in the case shown was sufficiently represented by Young's modulus) and the plastic irreversible strain component using Eq. (2).

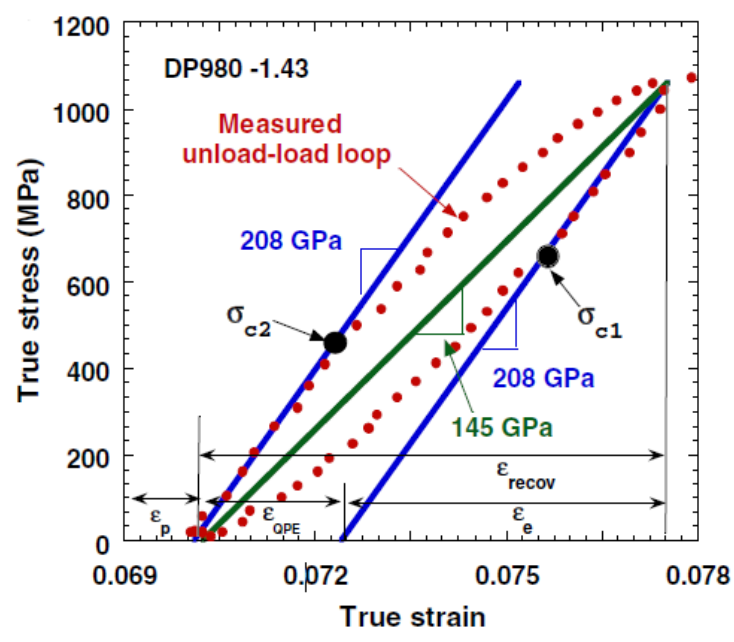

Figure 1. Expanded view of the unload-reload cycle for DP980 steel showing conceptual components of strain. After [4]

In the QPE constitutive model, the slope of the transitional stress-strain curve in uniaxial tension is represented as follows:

$E(\Delta \varepsilon)=\left\{\begin{array}{cc}E_{0} & \text { for } \Delta \varepsilon \leq \varepsilon_{c} \\ E_{0}-A_{1}\left[1-\exp \left\{-A_{2}\left(\Delta \varepsilon-\varepsilon_{c}\right)\right\}\right] & \text { for } \Delta \varepsilon \geq \varepsilon_{c}\end{array}\right.$

where $\mathrm{E}_{0}$ is the physical Young's modulus (or can be taken as an undetermined parameter), $\varepsilon_{\mathrm{c}}$ is a critical transition strain separating the linear and nonlinear regions, $\Delta \varepsilon$ is the absolute value of the increment of strain from the strain rate reversal, i.e., the unloading strain, and $A_{1}, A_{2}$ are material parameters. The equation has up to 4 undetermined parameters, i.e., $\mathrm{E}_{0}, \mathrm{~A} 1, \mathrm{~A}_{2}$ and $\varepsilon_{\mathrm{c}}$.

Integration of Eq. (3) leads to the equivalent stress form as follows:

$$
\Delta \sigma(\Delta \varepsilon)=\left\{\begin{array}{cc}
E_{0} \Delta \varepsilon & \text { for } \Delta \varepsilon \leq \varepsilon_{c} \\
\left(E_{0}-A_{1}\right)\left(\Delta \varepsilon-\varepsilon_{c}\right) & \\
+\frac{A_{1}}{A_{2}}\left\{1-\exp \left[-A_{2}\left(\Delta \varepsilon-\varepsilon_{c}\right)\right]\right\}+E_{0} \varepsilon_{c} & \text { for } \Delta \varepsilon \leq \varepsilon_{c}
\end{array}\right.
$$

where $\Delta \sigma$ is the absolute value of the stress drop during the unloading.

\subsection{Simple 1-Parameter representation}

In order to compare transition behavior in a wider range of alloys, it is highly advantageous to reduce the characterization of the nonlinearity to a single parameter. To do so, we make use of the simplifications noted above. In the current work, Eq. (1) is adopted with the small- 
strain / metal simplification, which we nickname the 1-P form (for one parameter):

$$
E_{T}(\varepsilon)=E_{0} \exp (-A \varepsilon)
$$

where $\mathrm{E}_{0}$ is taken here to be the independently-measured physical Young's modulus, and we call A the modulus reduction rate. $\mathrm{A}$ is a material constant representing the entirety of the transitional behavior for a single unloading leg. Note that this representation is a significant simplification of the more general QPE equation, Eq. 3. Although there is not space here to discuss it further, Chen et al. [9] recently showed that Eq. (5) adequately represents the material-to-material, approximately $80 \%$ of the variation represented by the 4-parameter Eq. 4. Therefore, it is useful for looking at broad comparisons.

Integration of Eq. (5) produces the equivalent stress form as follows:

$$
\Delta \sigma=\frac{\mathrm{E}_{0}}{\mathrm{~A}}[1-\exp (-\mathrm{A} \Delta \varepsilon)]
$$

\section{Experimental Procedures}

\subsection{Materials}

Twenty-six typical but diverse sheet forming alloys were tested. Tensile tests shown in Figure 2 illustrate the diverse properties in terms of tensile strength (213-1199 $\mathrm{MPa})$ and total elongation (5-49\%). Moreover, the assembled material set contains metals with a variety crystal structure, BCC, FCC and $\mathrm{HCP}$, and also a variety of hardening mechanisms and deformation mechanisms including slip, twinning (Mg, TWIP), and phase transformation (TRIP).

The "handbook" values of metals were adopted for following analyses as follows: $208 \mathrm{GPa}$ for steels, $70 \mathrm{GPa}$ for aluminium alloys, $110 \mathrm{GPa}$ for brass and titanium alloy, and $46 \mathrm{GPa}$ for magnesium alloys.

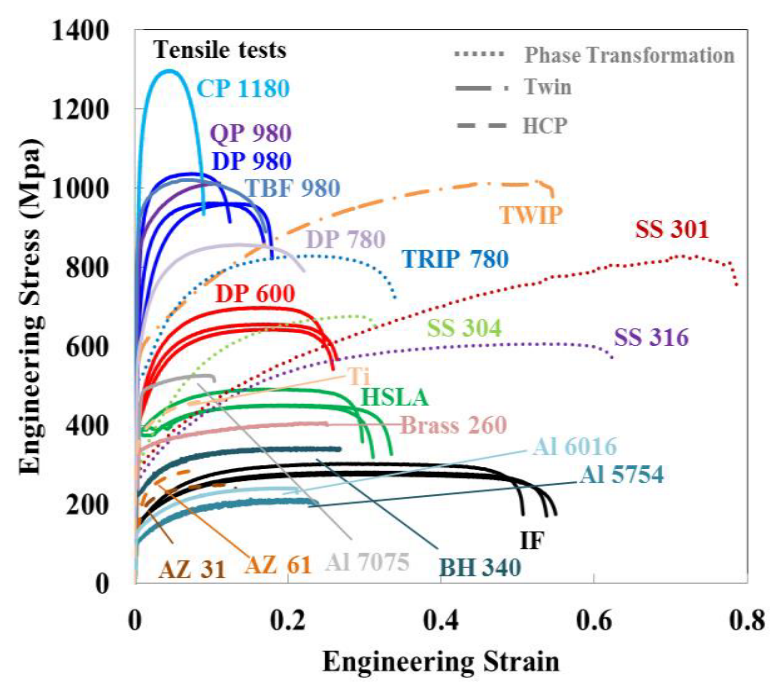

Figure 2. Tensile test results for 26 sheet alloys tested.

\subsection{Mechanical Test}

Note: For more information on testing and analysis procedures, refer to [9], upon which the current paper is largely based.

Standard ASTM E-8 tensile tests (RD) and unloading tests were carried out with an MTS 810 tensile frame. Strain was measured using a Class A Epsilon extensometer (3452-0200-030-ST), 2" gage, $+30 \% /-10 \%$ extension range. This extensometer is a commercial extensometer with the highest available resolution and accuracy. Data was collected at a sampling frequency of $10 \mathrm{~Hz}$ up to 0.005 strain and at $1 \mathrm{~Hz}$ for subsequent loading.

Tests were conducted at constant grip velocity of 0.08 $\mathrm{mm} / \mathrm{s}$, which is equivalent to nominal strain rate of $\sim 10^{-}$ $3 / \mathrm{s}$ and at room temperature. Two or three duplicated tests were conducted for core 12 steels $^{\mathrm{a}}$ to measure the test-totest scatter.

Similar tests aimed to characterize the transition behaviors were conducted with 5 LUL cycles at 2, 4, 6, 8 and $10 \%$ in engineering strain. Among them, only the unloading-loading cycle at $8 \%$ prestrain was used in the current work. Each instantaneous tangential slope point $\mathrm{E}_{\mathrm{T}}(=\mathrm{d} \Delta \sigma / \mathrm{d} \Delta \varepsilon)$ was taken as the mean value of the 6 adjacent $(\Delta \varepsilon, \Delta \sigma)$ data points. Other alternatives were considered to convert stress-strain data to slope-strain, but the procedure above led to reasonable combination of resolution and scatter for most experiments.

\section{Results and Analysis}

\subsection{Individual Fits of Unloading Legs}

In order to probe the extent of the linear region (i.e. the proportional limit), two versions of the QPE form, Eqs. (3) and (4), were utilized with the data for the 12 core steels. "QPE-4" refers to the 4 parameters being optimized. "QPE-3" eliminates $\varepsilon_{\mathrm{c}}$ by setting it equal identically to zero, thereby eliminating any linear region from the stress-strain representation (or, equivalently, any region of constant $\mathrm{E}_{\mathrm{T}}$ ).

The commercially software, Origin 8 SR3, was used to obtain optimized parameters by minimizing the leastsquares error relative to unloading stress-strain data $(\Delta \varepsilon$, $\Delta \sigma)$. All data was used in the optimization although a subset of all data points is shown in the following figures, for clarity of presentation. The optimized equation was then differentiated to obtain a corresponding tangential modulus, $\mathrm{E}_{\mathrm{T}}$, as a function of $\Delta \varepsilon$. Note that for this procedure the $\mathrm{E}_{\mathrm{T}}$ representation are not precisely optimal, but they are very similar.

The process and results are illustrated in Figure 3 along with the standard error of fit, $\langle\Delta \sigma\rangle$. Figure 3 (a) compares QPE-3 and QPE-4 fits with experimental unloading data for one core steel, DP980-1. A remarkable improvement, by a factor of 10 to 30 , is obtained by QPE

\footnotetext{
${ }^{\text {a }} 12$ core steels represents 4 DP980 steels, 4 DP600 steels, 4 HSLA steels, and 4 IF steels.
} 
fits as compared to the handbook physical or chord moduli. Figure 3 (b) shows $\mathrm{E}_{\mathrm{T}}$ vs. $\Delta \varepsilon$ plot using the same fit equation as used in Figure 3 (a). The results reveal that the QPE fits (to stress-strain) also reproduce the $\mathrm{E}_{\mathrm{T}}$ data better than the scatter of repeated tests with improvement by factors of $\sim 8$ and $\sim 4$, compared to handbook physical and chord moduli, respectively.

Most significantly, as can be seen in Figure 3 (a) and (b), the QPE-3 and 4 fits gave identical results! (The two lines overlay one another, so that only one can be seen.) That is, the optimal value of $\varepsilon_{c}$ in the QPE-4 fits was found to be 0 , such there is no distinction between the QPE-3 and QPE-4 forms. Therefore, no significant linear elastic region can be discerned for DP980-1. To make this more precise, the QPE-4 fits for 8 of the 12 core steels produced $\varepsilon_{\mathrm{c}}=0$. For the other 4 alloys, very small values of $\varepsilon_{\mathrm{c}}$ were determined, but none produced significant improvement of $\langle\Delta \sigma\rangle$. Therefore, it was concluded that the high-precision data available in this study suggests that the proportional limit is $0 \mathrm{MPa}$. That is, nonlinearity starts at the smallest stress excursions. This upsets nearly all standard teaching and learning on this subject.

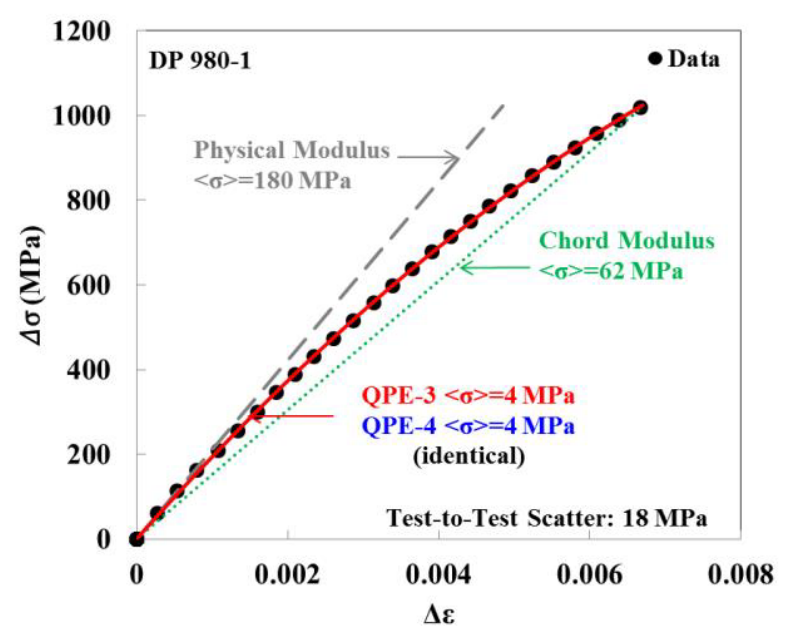

(a)

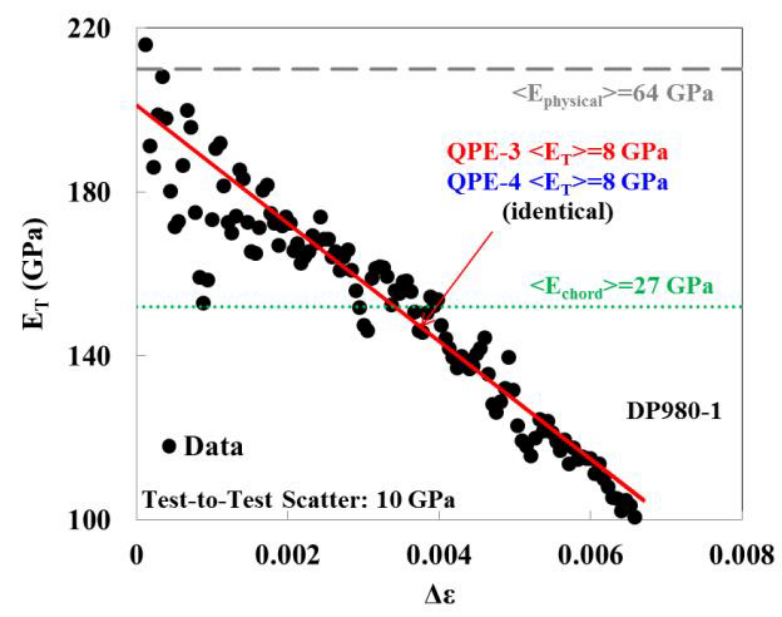

(b)

Figure 3. Comparison of QPE-3 and 4 for DP 980-1, (a) $\Delta \varepsilon$ vs. $\Delta \sigma$ and (b) $\Delta \varepsilon$ vs. $\mathrm{E}_{\mathrm{T}}$
As noted above, for use in comparing transitional behavior among various alloys the 1-P form is preferable. In order to test the ability of this simpler form to capture the essential transitional behavior, optimized 1-P fits were compared with the optimized QPE-3/4 fits, which are now known to reproduce the experimental precisely.

Figure 4 shows that for the same data as in Figure 3 (b), the 1-P fits introduce an insignificantly higher standard error of fit. It is insignificant because it remains lower than the test-to-test scatter for the tests upon which the analysis is based.

The fitting result for DP980-1 by 1-P in $\mathrm{E}_{\mathrm{T}}$ vs. $\Delta \varepsilon$ base is shown in Figure 4 together with results by QPE-3 and 4 fits. The 1-P fit leads to slightly higher standard deviation than QPE-3 and 4, but remains less than the test-to test scatter ( $9 \mathrm{GPa}$ vs. $10 \mathrm{GPa}$ ). This is particularly convincing in view of the fact that the 1-P fit is derived from stress-strain data, it was not optimized to the tangential modulus - strain result.

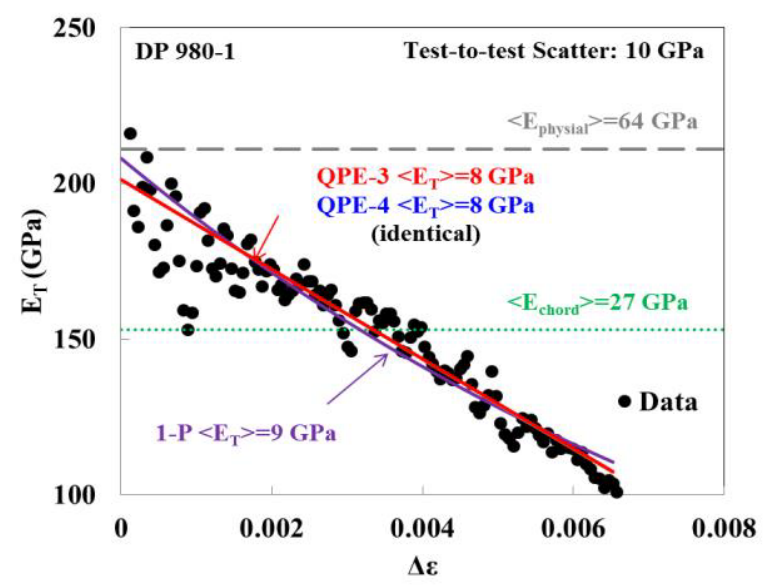

Figure 4. Comparison of fit equations for DP980-1

The corresponding analyses and comparison for the 26 materials are shown in Table 1. The results clearly reveal the tremendous improvements by any of the nonlinear equations compared with linear representations, i.e., either standard handbook or chord moduli. As expected, the 1-P fit leads to slightly higher standard error, but again remain less than the test-to-test scatter of the data itself. Based on the results, the use of 1-P form was deemed justified for further work.

Table 1 Summary of optimization using 1-P equation for 26 metals

\begin{tabular}{|c|c|c|}
\hline & $<\sigma>(\mathrm{MPa})$ & $<\mathrm{E}_{\mathrm{T}}>(\mathrm{GPa})$ \\
\hline $\mathrm{E}_{\text {physicl }}$ & 72 & 41 \\
\hline $\mathrm{E}_{\text {chord }}$ & 25 & 19 \\
\hline $1-\mathrm{P}$ & 5 & 8 \\
\hline QPE-3P & 2 & 6 \\
\hline QPE-4P & 2 & 6 \\
\hline Test-to-test scatter $^{\mathrm{b}}$ & 10 & 11 \\
\hline
\end{tabular}

${ }^{\mathrm{b}}$ The test-to-test scatter is calculated from duplicated data for 12 core steels only. 


\subsection{A Universal Law}

The simple 1-P representation, in view of the Sun and Wagoner and Chen observation that the transitional behavior in steels depends only on strength, offers the intriguing possibility of representing the entire phenomenon with zero fit parameters. If justified, this would render application of this significant improvement without any testing at all. (Recall, that the 1-P form improved the accuracy by factors of 4-8 as compared with the standard choices: zero-parameter Young's modulus or the 1-parameter chord modulus.)

In order to probe this idea, first a broader range of 21 steels was considered, including all of those shown in Table 1. These steels show the full range of hardening and deformation mechanisms and two of the three typical metal crystal structures, fcc and bcc. For this work we also included results for all pre-strains that were available.

Figure 5 (a) plots the modulus reduction rate $\mathrm{A}$ in the individual 1-P fits as a function of material strength ${ }^{\mathrm{C}}$ obtained from hundreds of loading-unloading-loading tests. The data shows a clear trend of a first-order dependency of modulus reduction rate on the material strength. The modulus reduction rate was found to fit the data by a curve as follows:

$$
A=\frac{15000 \mathrm{MPa}^{3 / 4}}{\sigma_{u}^{3 / 4} \mathrm{MPa}^{3 / 4}}
$$

The variation in $\mathrm{A}$ is captured within $<\mathrm{A}>=39$ (as compared with a total variation of 350) by using only the independently determined "strength," measuring anything with respect to the stress-strain transition.

The foregoing analysis was extended to all 26 tested materials with other base elements and crystal structures. Closer examination revealed that the A values for the other 5 metals exhibit same trend, i.e., a similar dependence on the unloading stress. However, the comparison also showed changes of A proportional to the physical Young's modulus for each metal. For instance, the A value for Brass 260 is approximately 100 whereas the A value for a steel of the same strength is approximately 200 , for a ratio is $1: 2$, approximately the same as the ratio of the physically measured Young's moduli for brass and steel: $110 \mathrm{GPa} / 208 \mathrm{GPa}$.

Using this second observed dependence of the modulus reduction rate on Young's modulus, the following "Universal Law" was derived taking into account both independently measured quantities, strength and Young's modulus:

$$
\begin{aligned}
& \Delta \sigma=\frac{208(G P a)\left[\sigma_{u}(M P a)\right]^{3 / 4}}{15000(M P a)^{3 / 4}} \\
& {\left[1-\exp \left(\frac{-15000(M P a)^{3 / 4}}{\left[\sigma_{u}(M P a)\right]^{3 / 4}} \frac{E_{0} G P a}{208 G P a} \Delta \varepsilon\right)\right]}
\end{aligned}
$$

$$
E=E_{0}(G P a) \exp \left(-\frac{15000(M P a)^{3 / 4}}{\left[\sigma_{u}(M P a)\right]^{3 / 4}} \frac{E_{0} \mathrm{GPa}}{208 G P a} \Delta \varepsilon\right) \text { (9) }
$$

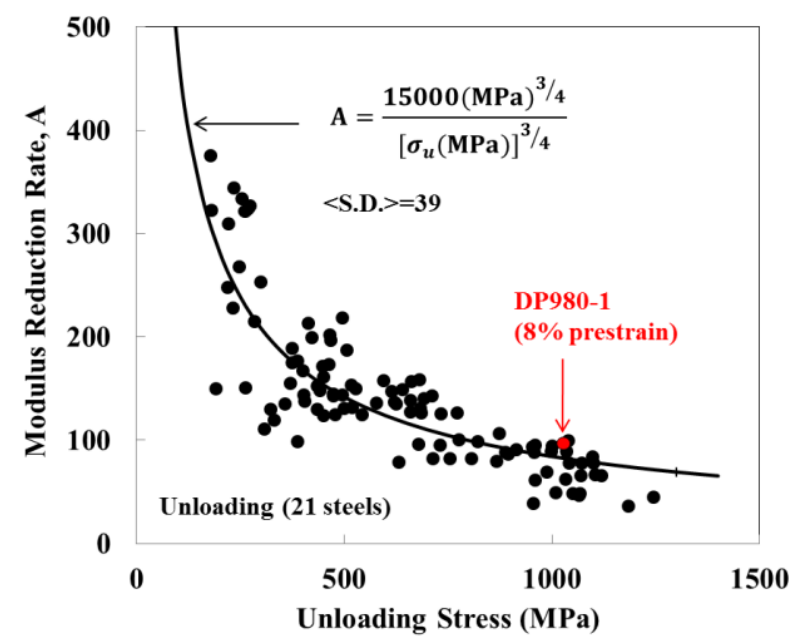

(a)

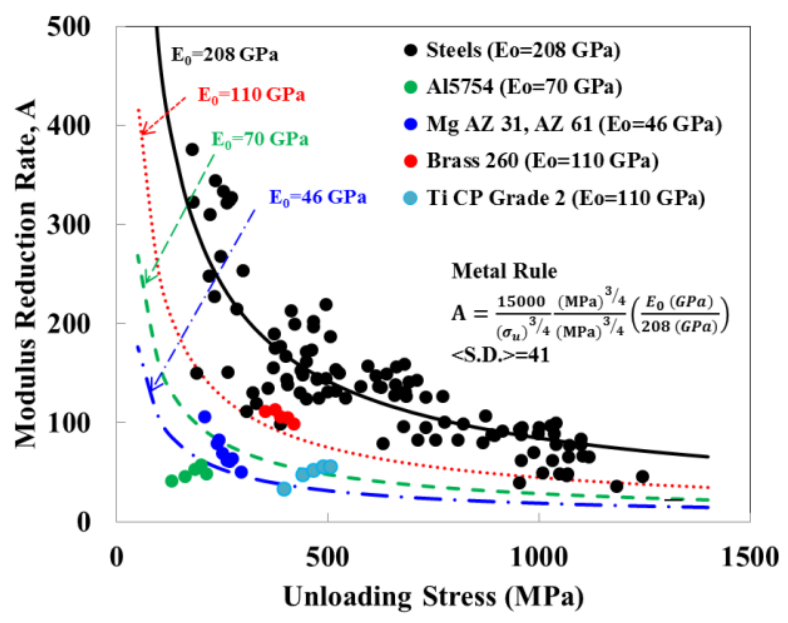

(b)

Figure 5. The variation of modulus reduction rate $\mathrm{A}$ vs. Unloading stress for all 26 metals (a) measured modulus reduction rates, A (b) modulus reduction rates, A, compared with a metal rule

The Universal Law is identical to the rules found for 12 steels or 21 steels, all of which had handbook Young's moduli of approximately $208 \mathrm{GPa}$.

To illustrate the use of the new relationship, the case of DP 980 with a pre-strain of 0.077 was again considered. Figure 6 compares the zero-parameter Universal Law with the custom 1-P fit for that data alone. The results show that there is only a slight loss of accuracy by using Eq. (9) compared with the 1-P fit (from $\left\langle\mathrm{E}_{\mathrm{T}}>=7\right.$ to $\left\langle\mathrm{E}_{\mathrm{T}}>=12 \mathrm{GPa}\right.$ ). Moreover, the zeroparameter Universal Law reduces the standard error relative to the 1-parameter chord modulus by a factor of greater than 2, i.e., $<\mathrm{E}_{\mathrm{T}}>=12$ to $27 \mathrm{GPa}$.

\footnotetext{
${ }^{\mathrm{c}}$ Throughout the current work, the material strength is measured by the flow stress just prior to unloading, $\sigma_{\mathrm{u}}$.
} 


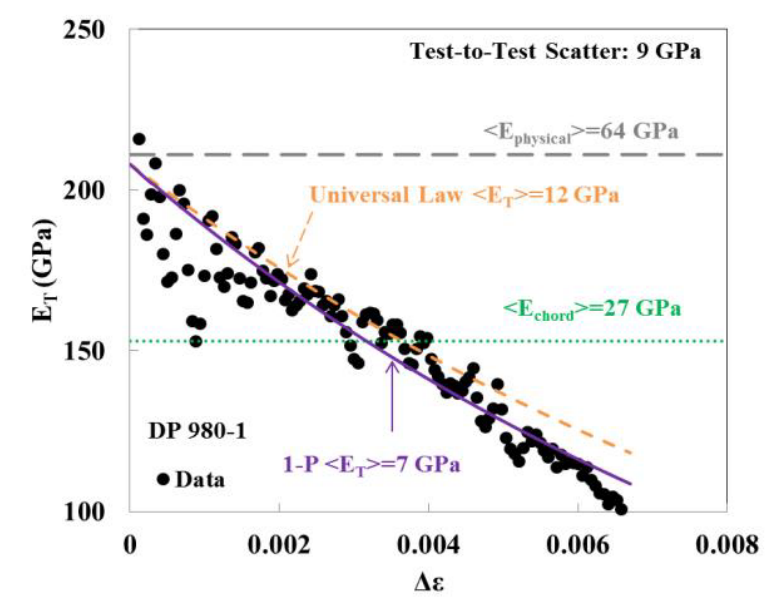

Figure 6. A comparison of $1-\mathrm{P}$ and the Universal Law for DP980-1

The accuracy of Eqs. (8) and (9) as compared with the 1-P custom fit for all 26 metals is compared in terms of average standard errors of fit in Table 2. The results reveal that the Universal Law can be used profitably in applications. It exhibits little or no loss of accuracy compared with test-to-test scatter. Moreover, it greatly improves on the commonly-used linear representations, i.e., physical Young' modulus or chord modulus. Furthermore, it does not require any special test to implement profitably.

Table 2. Comparison of Universal Law and custom 1-P fit for 26 metals

\begin{tabular}{|c|c|c|}
\hline & $<\sigma>(\mathrm{MPa})$ & $<\mathrm{E}_{\mathrm{T}}>(\mathrm{GPa})$ \\
\hline $\mathrm{E}_{\text {physicl }}$ & 72 & 41 \\
\hline $\mathrm{E}_{\text {chord }}$ & 25 & 19 \\
\hline Universal Law & 13 & 11 \\
\hline $1-\mathrm{P}$ & 5 & 8 \\
\hline Test-to-test scatter & 10 & 11 \\
\hline
\end{tabular}

Using the Eqs. (8) and (9), the hysteresis loop width, $\delta$, can be derived as follows:

$$
\begin{gathered}
\delta=\frac{2}{A} \ln \left[1-\frac{A}{E_{0}} \sigma_{u}+\frac{A}{E_{0}}\left(\sigma_{u}-\frac{E_{0}}{A}-\frac{\sigma_{u}}{\ln \left(1-\frac{A \sigma_{u}}{E_{0}}\right)}\right)\right] \\
\left.-\frac{\sigma_{u}-\left(\sigma_{u} \frac{E_{0}}{A}-\frac{\sigma_{u}}{\ln \left(1-\frac{A \sigma_{u}}{E_{0}}\right)}\right)}{\sigma_{u}}\right] \ln \left(1-\frac{A}{E_{0}} \sigma_{u}\right)
\end{gathered}
$$

A broad comparison of measured and Universal-Lawpredicted loop widths is shown in Figure 7 for all 27 materials tested. Figure 7 (a) shows the predicted loop width from the Universal Law together with measured loop width as a function of the unloading stress. The results are in good agreement except for two steels which exhibit exceptionally small hysteresis relative to the trends of the other alloy. In order to assess realistically how good the agreement is, please note that either of the current standard approaches, Young's modulus or chord modulus, exhibit NO loop width. That is, plotting those data would put them all on the X-axis of Figure 7 (a).

Figure 7 (b) directly compares measured and predicted loop width. Although there is some systematic deviation, the Universal Law represents the highlysensitive loop-width data within the scatter of the data itself, with the possible exception of two "special" steels, i.e., CP 1180 and TBF 980. These metals showed (almost) linear-like unloading behavior, with less reduction of the modulus than for the other alloys. These two alloys are known to have extremely fine microstructures, which suggest a possible mechanism for this behavior and their divergence from the norms. This will be discussed in Section 5 .

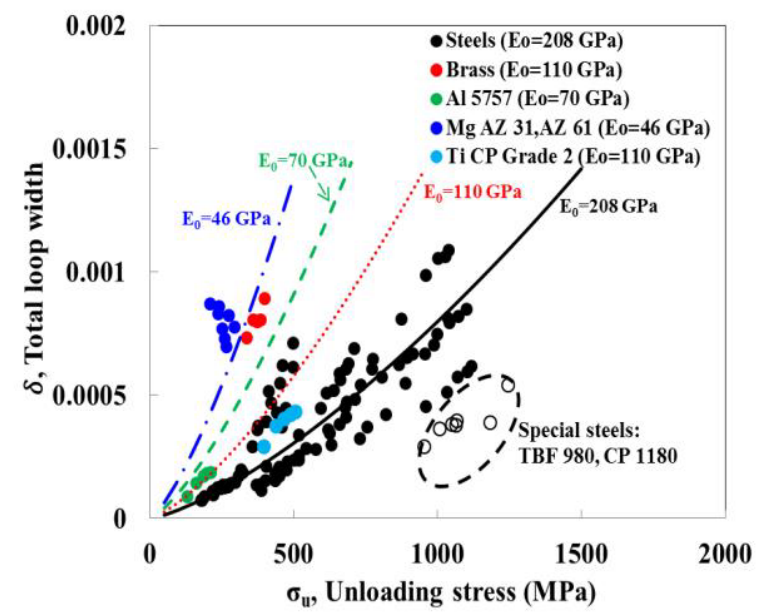

(a)

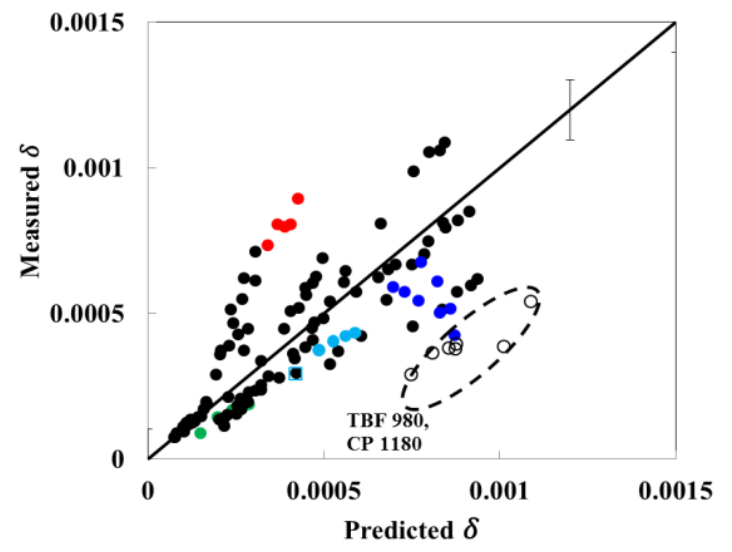

(b)

Figure 7 Summary of loop width $(\delta)$ measurements at various pre-strains compared with Universal Law predictions: (a) as a function of unloading stress and Young's modulus, and (b) compared with Universal Law predicted values. 


\section{Application}

The Universal Law was applied to Toyota pillar compression tests, as are presented in detail elsewhere [8]. $600 \mathrm{~mm}$ long structural pillars was formed in two pieces from SPC 400 steel sheet $(0.8 \mathrm{~mm}$ thick steel cold-rolled with an ultimate tensile strength of $440 \mathrm{MPa}$ ) and spot welded. The welded pillars were then compressed in a universal testing machine with behavior as shown in Figure 8 .

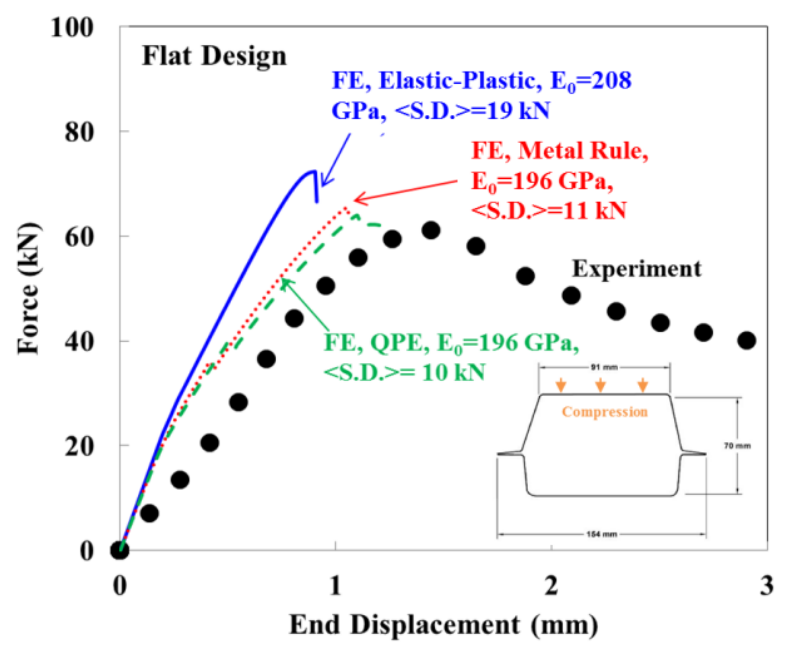

Figure 8. Toyota compression test data and FE prediction by QPE equation and Universal Law

The pillar compression was also simulated using Abaqus/Standard incorporating either the QPE-4 form or the Universal Law. For QPE-4, a custom value of $\mathrm{E}_{0}$ obtained by ultrasonic testing was adopted. In keeping with the simplest application, the Universal Law was applied using the handbook value of Young's modulus.

The predicted force-displacement curves are compared in Figure 8 with the experimental data. The advanced models, i.e., QPE-4 and Universal Law, improve the prediction accuracy compared with the standard FE result as a factor of $\sim 2$. Moreover, the Universal Law applied with no adjustable parameters produces almost identical fidelity as compared with 4paramter QPE-4. This application confirms the usefulness of the Universal Law for engineers who need to accurately predict springback or automotive structural crush without incurring a corresponding time and effort for additional experiments.

\section{Discussion}

Many mechanisms have been proposed as accounting for the modulus effect in metals, whether it is considered a linear departure from the physical Young's modulus, or a nonlinearity. The current work suggests an answer to the question of "why?"

The simplest proposal is second-order nonlinear elasticity related to bond stretching [10]. As already discussed, the well-established energy absorption and nonlinearity as vanishingly small strains as confirmed here rules out this mechanism.
A twinning and detwinning [11-13] mechanism cannot explain experimentally observed nonlinearity in non-HCP structured metals.

Textural changes during the plastic pre-straining $[13,14]$ can be also considered. However, these changes occur too slowly with strain to account for the observed changes. Furthermore, textural changes would not be expected to introduce nonlinearities, and would be expected to lead to higher modulus changes at higher stain in many cases, which is never observed.

Material damage by porosity or void evolution has been also proposed $[15,16]$. However, the reduction of density by the damage mechanism was found as insufficient to explain experimentally observed modulus degradation [17].

Heterogeneity arising from internal and residual stresses $[13,14,18]$ established by microstructural inhomogeneities during pre-straining can cause additional micro-strain or stress in the subsequent unloading. However, these effects would not be expected to reverse during an unloading leg with no plastic deformation.

The mechanism that seems the most plausible on the surface is a generalized dislocation pile-up and relaxation mechanism $[2,19,20]$. Dislocations are known to interact elastically with one another but also dissipate energy whenever they move [20]. Thus, the main features are satisfied. In this concept, mobile dislocations move on slip planes until stopped by obstacles such as grain boundary or inclusions. When the external force is removed, the dislocations repel each other and provide additional micro-strain concurrent with elastic unloading strain. Such a mechanism reproduces all of the qualitative features observed. For example, all moving dislocations dissipate work by exciting lattice phonons [21].

The two "special" steels, CP 1180 and TBF 980, having abnormally small loop widths and are known to have very fine microstructures as is beneficial for increasing flow strength. However, extremely fine microstructures also introduce the constraint of extremely limited pile-up lengths, which can potentially limit the storage and release of strain.

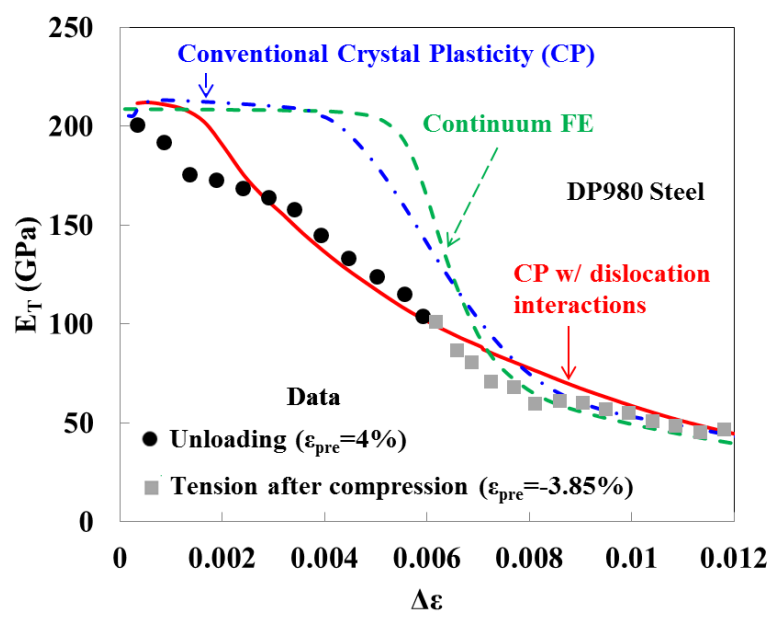

Figure 9. Comparison of the measured tangential modulus with FE simulations incorporating alternate constitutive models. 
As part of other, so far unpublished work, the authors simulated unloading and reverse loading (i.e. unloading and then compression after a tensile prestrain) using a carefully-constructed RVE based on a DP980 steel microstructure. Care was taken to use the proper microstructural properties, sizes and proportions in the RVE. Figure 9 shows the compares the measured tangential slope change to simulations using three constitutive models: a standard Continuum elastic-plastic model, a standard Crystal Plasticity (CP) model, and CP model with dislocation elastic interactions [22-24]. The results suggest that the dislocation pile-up and relaxation mechanism dominates the nonlinear transition behavior.

\section{Conclusions}

The elastic-plastic transition behavior of 26 metals was analysed using advanced nonlinear models and hundreds of special tensile test data using a Class A extensometer. Based on the analyses and observations, following conclusions were reached:

- A zero-parameter Universal Law predicting the transitional stress-strain response in all metals with no undetermined parameters was introduced.

- Implementing the Universal Law requires no testing but was shown to improve the accuracy of simulating automotive crush, by a factor of two.

- The proportional limit of metals is $0 \mathrm{MPa}$. That is, nonlinearity starts for vanishingly small strains.

- A simple 1-parameter equation describes the transition response well for all transition regions, without significant loss of accuracy compared with multiparameter models appearing in the literature.

- The dominant mechanism of transitional behavior appears to be a dislocation pile-up and release.

\section{Acknowledgements}

This material is based upon work supported by the U.S. Department of Energy, Office of Science, Office of Basic Energy Sciences, Materials Science and Engineering Division, and particularly Program Director John S. Vetrano, for their support of the current work under Award Number DE-SC0012483 and DE-SC0012587. The 12 core steels were provided by ArcelorMittal, Nucor, ThyssenKrupp, and U. S. Steel, as arranged by the Steel Marketing and Development Institute. Six other steels were provided by Posco.

\section{References}

1. F. Morestin, M. Boivin, Nucl. Eng. Des. 162, 107-16 (1996)

2. R. Cleveland, A. Ghosh, Int. J. Plast. 18, 769-85 (2002)

3. F. Yoshida, T. Uemori, K. Fujiwara, Int. J. Plast. 18, 633-59 (2002)

4. L. Sun, R.H. Wagoner, Int. J. Plast. 27, 1126-44 (2011)
5. A.S. Argon, Physical Metallurgy. (Elsevier; 1996)

6. J. H. Rose, J.R. Smith, J. Ferrante, Phys. Rev. B. 28, 1835-45 (1983)

7. H. Zhu, S. Sriram, B. Yan, P. Duroux, SAE Int. J. Mater. Manuf. 3, 691-701 (2010)

8. Z. Chen, U. Gandhi, J. Lee, R.H. Wagoner, J. Mater. Process. Technol. 227, 227-43 (2016)

9. Z. Chen, H.J. Bong, D. Li, R.H. Wagoner, Int. J. Plast. (in final review)

10. B.E. Powell, J. Appl. Phys. 53, 764 (1982)

11. C. Cáceres, T. Sumitomo, M. Veidt, Acta. Mater. 516, 211-8 (2003)

12. T. Hama, N. Kitamura, H. Takuda, Mater. Sci. Eng. A. 583, 232-41 (2013)

13. H. Wang, P.D. Wu, J. Wang, Int. J. Plast. 47, 49-64 (2013)

14. S.R. Agnew, J.R. Weertman, Mater. Sci. Eng. A. 242, 174-80 (1998)

15. S. Murakami, Continuum damage mechanics (Springer, 2012)

16. K. Yamaguchi, H. Adachi, N. Takakura, Met. Mater. Int. 4, 420-5 (1998)

17. M. Yang, Y. Akiyama, T. Sasaki, J. Mater. Process. Technol. 151, 232-6 (2004)

18. A. Govik, R. Rentmeester, L. Nilsson, Mater. Sci. Eng. A. 602, 119-26 (2014)

19. J.H. Kim, D. Kim, F. Barlat, M.-G. Lee, Mater. Sci. Eng. A. 539, 259-70 (2012)

20. L. Luo, A.K. Ghosh, J. Eng. Mater. Technol. 125, 237 (2003)

21. J.P. Hirth, J. Lothe. Theory of Dislocations (Krieger Publishing Company, 1982)

22. M.-G. Lee MG, H. Lim, B.L. Adams, J.P. Hirth, R.H. Wagoner, Int. J. Plast. 20, 925-38, (2010)

23. H. Lim, M.-G. Lee, J.H. Kim, B.L. Adams, R.H. Wagoner, Int. J. Plast. 27, 1328-53 (2011)

24. H. Lim, S. Subedi, D.T. Fullwood, B.L. Adams, R.H. Wagoner, Mater Trans, 55, 35-8, (2014) 